Supporting Information for

\title{
Experimental and theoretical study of gamma radiolysis and dose rate effect of $o$-cresol formaldehyde epoxy composites
}

\author{
Qiang Liu, Wei Huang, Bo Liu, Pu-cheng Wang ${ }^{*}$, Hong-bing Chen ${ }^{\dagger}$
}

Institute of Nuclear Physics and Chemistry, China Academy of Engineering Physics, Mianyang 621000, China

smilingwind@foxmail.com

hongbing2014@foxmail.com 
Table S 1 Relationships between monomer ratios (phenolic hydroxyl to epoxide) and glass transition temperatures.

\begin{tabular}{cc}
\hline Ratio & Glass transition temperature $\left({ }^{\circ} \mathrm{C}\right)$ \\
\hline 0.2 & 162 \\
0.6 & 178 \\
0.7 & 186 \\
1.0 & 153 \\
\hline
\end{tabular}

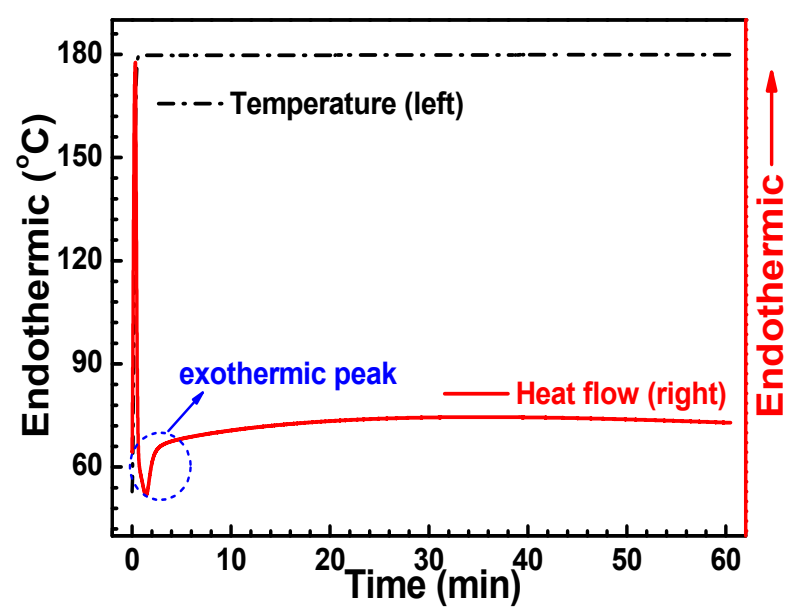

Figure S 1 Isothermal thermogram of epoxy and $p$-tert-butyl phenyl formaldehyde system. The isothermal curing was performed by heating the sample from $50{ }^{\circ} \mathrm{C}$ to $180{ }^{\circ} \mathrm{C}$ at a rate of $300^{\circ} \mathrm{C} / \mathrm{min}$ in $\mathrm{N}_{2}$ atmosphere, and then kept at $180^{\circ} \mathrm{C}$ for $60 \mathrm{~min}$.
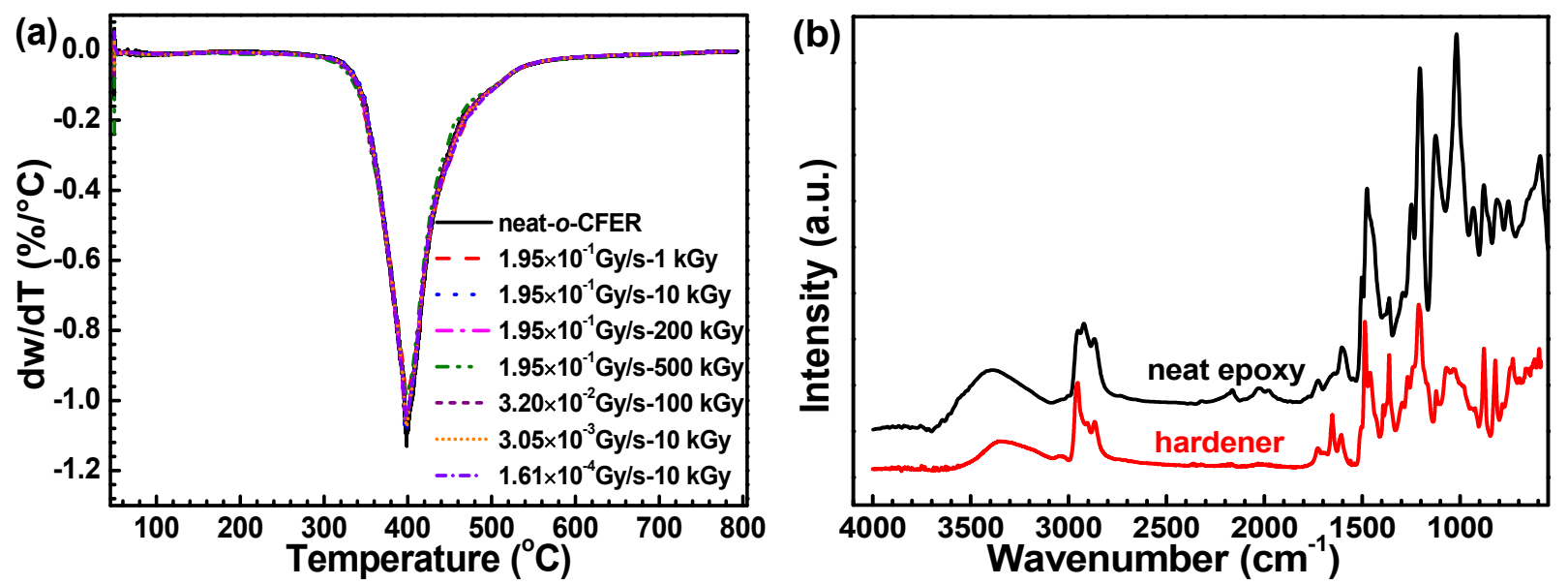

Figure S 2 (a) Differential curves of the weight loss curves of the neat and $\gamma$ radiated $o$-CFER resins, (b) FTIR spectra of the neat $o$-CFER and hardener. 
Table S 2 Assignment and nature of the absorption peaks in the FTIR spectra of $o$-CFER. ${ }^{1-12}$

\begin{tabular}{|c|c|c|c|}
\hline $\begin{array}{l}\text { Wavenumber } \\
\qquad\left(\mathrm{cm}^{-1}\right)\end{array}$ & Assignment and nature & $\begin{array}{l}\text { Wavenumber } \\
\qquad\left(\mathrm{cm}^{-1}\right)\end{array}$ & Assignment and nature \\
\hline $3100-3680$ & $\begin{array}{c}\text { alcoholic } \mathrm{OH}(3420) \\
\text { hydrogen-bonded } \mathrm{OH}(3361) \\
\text { phenolic } \mathrm{OH}(3394) \\
\text { secondary amine } v_{\mathrm{N}-\mathrm{H}}(3300-3600)\end{array}$ & 3109 & $\begin{array}{l}\text { stretching vibration of } \\
\text { associated } \mathrm{C}=\mathrm{N}-\mathrm{H} \text { in the } \\
\text { imine }\end{array}$ \\
\hline 2955 & $v_{\text {as C-H }}$ of $\mathrm{CH}_{3}$ & 2921 & $\begin{array}{c}v_{\text {as C-H }} \text { of }-\mathrm{CH}_{2-} \\
\text { Acetone/chain ketone } \\
(1708)\end{array}$ \\
\hline 2868 & $v_{\mathrm{s}-\mathrm{H}}$ of $-\mathrm{CH}_{3}$ and $-\mathrm{CH}_{2}-$ & 1731 & $\begin{array}{l}\text { carboxylic acid (1735) } \\
\text { methyl ketone/aldehyde } \\
\qquad(1722) \\
\text { formate (1738) }\end{array}$ \\
\hline $1680^{\mathrm{a}}$ & amide & 1668 & $\begin{array}{l}\text { stretching vibration of } \\
\qquad \mathrm{C}=\mathrm{N} \text { in imine }\end{array}$ \\
\hline $1660^{\mathrm{a}}$ & unsaturated amide & $1649-1652$ & $\begin{array}{l}\text { aromatic ketone/primary } \\
\text { amine/double } \\
\text { bonds/tertiary amide } / v_{\mathrm{O}-\mathrm{H}} \\
\text { of water }\end{array}$ \\
\hline $\begin{array}{c}1605,1501, \\
1450\end{array}$ & $\begin{array}{c}\text { vibration of the skeleton of benzene } \\
\text { ring }\end{array}$ & 1596 & $\begin{array}{l}\text { bending vibration of } \mathrm{N}-\mathrm{H} \\
\text { in } \mathrm{C}=\mathrm{N}-\mathrm{H} \text { (hardener) }\end{array}$ \\
\hline $1560-1580$ & ammonium carbonate & 1560 & carboxylate \\
\hline 1477 & $\delta_{\mathrm{C}-\mathrm{H}}$ deformation of aliphatic $-\mathrm{CH}_{2^{-}}$ & 1394,1376 & $\begin{array}{l}\text { splitting of }-\mathrm{C}\left(\mathrm{CH}_{3}\right)_{3} \\
\text { deformation of } \mathrm{CH}_{3}\end{array}$ \\
\hline 1362 & deformation in plane of phenolic $\mathrm{OH}$ & 1294 & $\begin{array}{c}-\mathrm{CH}_{2} \text { - twisting vibration } \\
\text { in imine }\end{array}$ \\
\hline
\end{tabular}




\begin{tabular}{|c|c|c|c|}
\hline 1249 & $v_{\mathrm{C}-\mathrm{O}}$ of ether $\left(\mathrm{C}-\mathrm{O}-\mathrm{Ph}^{\mathrm{b}}\right)$ & 1128 & isopropanol \\
\hline 807,931 & $v_{\mathrm{C}-\mathrm{O}-\mathrm{C}}$ of epoxide ring & $754-879$ & $\begin{array}{c}\delta_{\mathrm{C}-\mathrm{H}} \text { of substituted } \\
\text { benzene rings }\end{array}$ \\
\hline
\end{tabular}

${ }^{a}$ Non-existent peaks. ${ }^{b}$ Benzene ring.
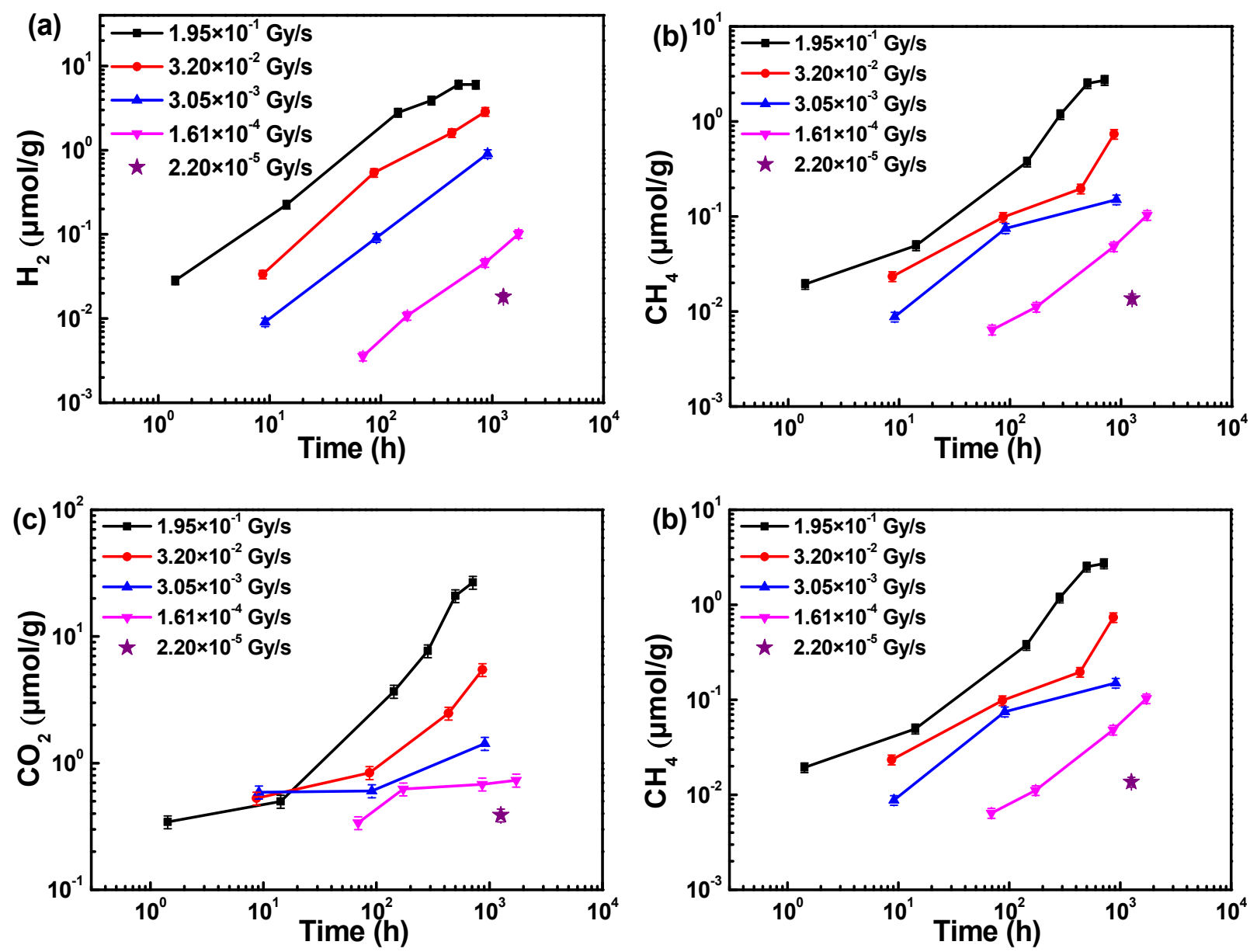

Figure S 3 Gas yields of (a) hydrogen, (b) methane, (c) carbon dioxide and (d) carbon monoxide with radiation time.

Table S 3 Logarithmic relative shift factor $\left(\ln \Delta \alpha_{T}\right)$ for hydrogen based on different reference conditions.

\begin{tabular}{|c|c|c|c|c|}
\hline \multirow{2}{*}{$\begin{array}{l}\text { Reference radiation } \\
\text { rates }(\mathrm{Gy} / \mathrm{s})\end{array}$} & \multicolumn{4}{|c|}{ Relative logarithmic shift factor $\left(\ln \Delta \alpha_{T}\right)$} \\
\hline & $1.95 \times 10^{-1}$ & $3.20 \times 10^{-2}$ & $3.05 \times 10^{-3}$ & $1.61 \times 10^{-4}$ \\
\hline $1.95 \times 10^{-1}$ & - & -1.46 & -2.92 & -5.82 \\
\hline $3.20 \times 10^{-2}$ & - & - & -1.46 & -4.36 \\
\hline $3.05 \times 10^{-3}$ & - & - & - & -3.00 \\
\hline
\end{tabular}


Table S 4 Logarithmic relative shift factor $\left(\ln \Delta \alpha_{T}\right)$ for methane based on different reference conditions.

\begin{tabular}{ccccc}
\hline $\begin{array}{c}\text { Reference radiation } \\
\text { rates }(\mathrm{Gy} / \mathrm{s})\end{array}$ & $1.95 \times 10^{-1}$ & $3.20 \times 10^{-2}$ & $3.05 \times 10^{-3}$ & $1.61 \times 10^{-4}$ \\
\hline $1.95 \times 10^{-1}$ & - & -1.47 & -2.70 & -4.53 \\
$3.20 \times 10^{-2}$ & - & - & -1.29 & -3.38 \\
$3.05 \times 10^{-3}$ & - & - & - & -2.53 \\
$1.61 \times 10^{-4}$ & - & - & - & - \\
\hline
\end{tabular}

Table S 5 Logarithmic relative shift factor $\left(\ln \Delta \alpha_{T}\right)$ for carbon dioxide based on different reference conditions.

\begin{tabular}{ccccc}
\hline $\begin{array}{c}\text { Reference radiation } \\
\text { rates }(\mathrm{Gy} / \mathrm{s})\end{array}$ & $1.95 \times 10^{-1}$ & $3.20 \times 10^{-2}$ & $3.05 \times 10^{-3}$ & $1.61 \times 10^{-4}$ \\
\hline $1.95 \times 10^{-1}$ & - & -1.43 & -2.82 & -5.49 \\
$3.20 \times 10^{-2}$ & - & - & -1.40 & -4.08 \\
$3.05 \times 10^{-3}$ & - & - & - & -2.72 \\
$1.61 \times 10^{-4}$ & - & - & - & - \\
\hline
\end{tabular}

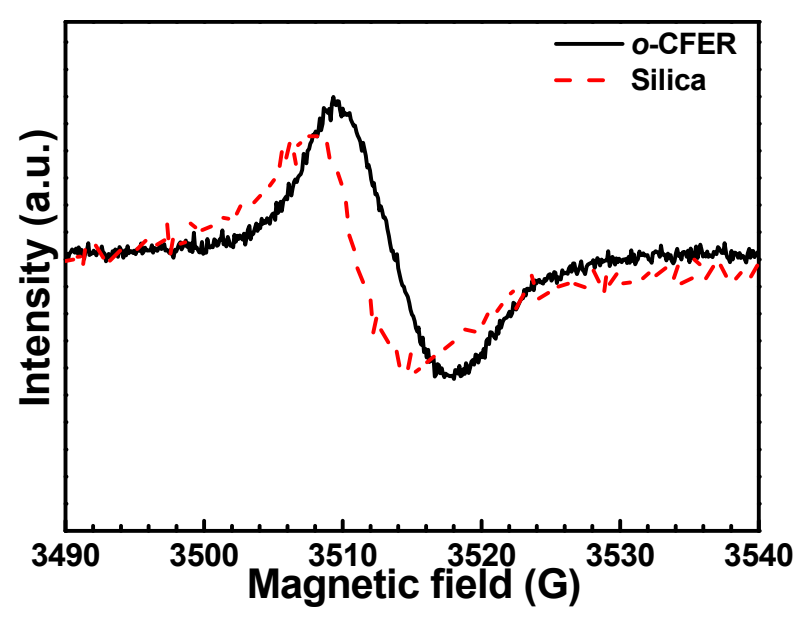

Figure S 4 Comparison of the EPR spectra of silica and $o$-CEFR resin.

Table S 6 Isotropic Fermi contact couplings (MHz). 


\begin{tabular}{|c|c|c|c|c|c|}
\hline \multicolumn{2}{|c|}{ Radical (d) ${ }^{\mathrm{a}}$} & \multicolumn{2}{|c|}{ Radical $(\mathrm{e})^{\mathrm{a}}$} & \multicolumn{2}{|c|}{ Radical (f) ${ }^{\mathrm{a}}$} \\
\hline $10 \mathrm{H}$ & 7.3577 & $35 \mathrm{H}$ & -17.9366 & $29 \mathrm{H}$ & 65.6670 \\
\hline $11 \mathrm{H}$ & -25.5500 & $36 \mathrm{H}$ & 5.2570 & $30 \mathrm{H}$ & 264.5841 \\
\hline $12 \mathrm{H}$ & 7.0064 & $37 \mathrm{H}$ & 6.6797 & $31 \mathrm{H}$ & 5.1826 \\
\hline $13 \mathrm{H}$ & 29.7920 & $39 \mathrm{H}$ & 7.6662 & $32 \mathrm{H}$ & 1.3203 \\
\hline $15 \mathrm{H}$ & 30.3158 & $44 \mathrm{H}$ & 7.8760 & $33 \mathrm{H}$ & 2.3489 \\
\hline $39 \mathrm{H}$ & 10.2129 & $49 \mathrm{H}$ & 1.9331 & & \\
\hline $40 \mathrm{H}$ & 7.4856 & $50 \mathrm{H}$ & 11.8052 & & \\
\hline
\end{tabular}

${ }^{\text {a }}$ Radical images and the atomic No. are displayed in Figure 10 of the manuscript.

\section{Reference}

(1) Djouani, F.; Zahra, Y.; Fayolle, B.; Kuntz, M.; Verdu, J. Degradation of epoxy coatings under gamma irradiation. Radiat. Phys. Chem. 2013, 82, 54-62.

(2) Longiéras, N.; Sebban, M.; Palmas, P.; Rivaton, A.; Gardette, J. L. Degradation of epoxy resins under high energy electron beam irradiation: Radio-oxidation. Polym. Degrad. Stab. 2007, 92 (12), 2190-2197.

(3) Devanne, T.; Bry, A.; Audouin, L.; Verdu, J. Radiochemical ageing of an amine cured epoxy network. Part I: change of physical properties. Polymer 2005, 46 (1), 229-236.

(4) Hou, L.; Wu, Y.; Shan, D.; Guo, B.; Zong, Y. Dose rate effects on shape memory epoxy resin during $1 \mathrm{MeV}$ electron irradiation in air. J. Mater. Sci. Technol. 2021, 67, 61-69.

(5) Li, Y. F.; Hu, S. L.; Zeng, Z. B.; Liu, X.; Xiang, W.; Xiao, M. Z. Effect of the Gamma-Ray Irradiation on the Properties of an Epoxy Encapsulant. Mater. Sci. Forum 2019, 944, 600-606.

(6) Chang, S.; Li, J.; Han, W.; Zhang, Z.; Chang, L.; Chen, W.; Li, Z.; Dai, Y.; Chen, D. Fabrication and high radiation-resistant properties of functionalized carbon nanotube reinforced novolac epoxy resin nanocomposite coatings. RSC Adv. 2016, 6 (63), 58296-58301.

(7) Wei, X.; Xue, H.; Wang, J.; Tao, W.; Li, S.; Hu, G.; Fan, X.; Hao, G.; He, J. Functionlized graphene serving as free radical scavenger and corrosion protection in gamma-irradiated epoxy composites. Carbon 2016, 101, 315-323.

(8) Hou, L.; Wu, Y.; Shan, D.; Bin, G.; Zong, Y. High energy proton irradiation stability and damage mechanism of shape-memory epoxy resin. Smart Mater. Struct. 2019, 28 (11), 115003.

(9) Rami, N.; Meghraoui, H.; Ziraoui, R.; Khoukhi, T.; Mouhib, M.; Elharfi, A. Influence of gamma irradiation on the chemical and physical properties of DGEDDS/PDA and DGEDDS/MDA epoxy resins. Journal of Materials and Environmental Science 2010, 1, 277-288.

(10) Musto, P.; Ragosta, G.; Abbate, M.; Scarinzi, G. Photo-Oxidation of High Performance Epoxy Networks: Correlation between the Molecular Mechanisms of Degradation and the Viscoelastic and Mechanical Response. Macromolecules 2008, 41 (15), 5729-5743.

(11) Morsch, S.; Liu, Y.; Lyon, S. B.; Gibbon, S. R.; Gabriele, B.; Malanin, M.; Eichhorn, K.-J. 
Examining the early stages of thermal oxidative degradation in epoxy-amine resins. Polym. Degrad. Stab. 2020, 176, 109147.

(12) Bahlakeh, G.; Ramezanzadeh, B. A Detailed Molecular Dynamics Simulation and Experimental Investigation on the Interfacial Bonding Mechanism of an Epoxy Adhesive on Carbon Steel Sheets Decorated with a Novel Cerium-Lanthanum Nanofilm. ACS Appl. Mat. Interfaces 2017, 9 (20), 17536-17551. 